\title{
LA CBT-E a distanza nei programmi di riabilitazione residenziale intensiva per i disturbi dell'alimentazione durante l'epidemia COVID-19
}

\author{
Marco Massa \\ Centro Terapeutico Villa del Principe, Genova, Italia
}

\section{Parole chiave}

Disturbi dell'alimentazione

Terapia cognitivo comportamentale CBT-E

Terapia a distanza

Telemedicina

Coronavirus

COVID-19

\begin{abstract}
Riassunto
La diffusione pandemica della malattia da coronavirus (COVID-19) ha spinto la maggior parte dei governi nel mondo a mettere in lockdown il proprio paese. Durante il lockdown le regole sul distanziamento sociale, sulla quarantena e sull'isolamento hanno reso molto complicata la somministrazione delle terapie psicologiche a tutti i livelli di cura. Nella nostra Unità di Riabilitazione Nutrizionale Intensiva di Villa del Principe (Genova - Italia) l'utilizzo di soluzioni di terapia a distanza per somministrare la terapia cognitivo comportamentale migliorata (CBT-E) è stata una scelta obbligata per garantire la continuità dei programmi di cura dei pazienti con disturbi dell'alimentazione. La CBT-E, anche nella sua forma intensiva applicata in regime di ricovero, si è dimostrata particolarmente adatta ad essere somministrata online in alcune sue parti. In questo lavoro sono prese in esame le procedure della CBT-E intensiva residenziale che hanno subito il maggiore impatto dalle misure anti COVID-19 e come tali difficoltà siano state superate attraverso I'utilizzo di soluzioni a distanza.
\end{abstract}

\section{Ringraziamenti}

Ringrazio Arianna Banderali (Villa Garda) e Lorenzo Maura (Villa del Principe) per il loro utile contributo a questo articolo. Vorrei anche ringraziare i miei colleghi di Villa del Principe (Genova) per il prezioso supporto nello sviluppo delle procedure terapeutiche descritte in questo lavoro.

\begin{abstract}
The pandemic spread of coronavirus disease (COVID-19) has forced most governments around the world to put their country on lockdown. During the lockdown, the rules on social distancing, quarantine, and isolation made the delivery of psychological therapies very complicated at all levels of care. In our Intensive Nutritional Rehabilitation Unit of Villa del Principe (Genoa - Italy) the use of remote therapy solutions to deliver enhanced cognitive behavior therapy (CBT-E) was a forced choice to guarantee the continuity of care for patients with eating disorders. CBT-E, even in its intensive form applied to inpatients, has proved particularly suitable to be delivered remotely in some of its parts. This paper examines the CBT-E procedures (for inpatients) that have undergone the greatest impact from the antiCOVID-19 measures and how these difficulties have been overcome through the use of remote solutions.
\end{abstract}

\begin{abstract}
Acknowledgements
I would like to acknowledge Dr Arianna Banderali (Villa Garda) and Dr Lorenzo Maura (Villa del Principe) for their helpful contribution to this article. I also would like to thank my collegues of Villa del Principe (Genoa) for the precious support in developing the therapeutic procedures described in this paper.
\end{abstract}

Copyright @ 2020 Marco Massa. This is an open-access article distributed under the terms of the Creative Commons Attribution License (CC BY). The use, distribution or reproduction in other forums is permitted, provided the original author(s) and the copyright owner(s) are credited and that the original publication in this journal is cited, in accordance with accepted academic practice. No use, distribution or reproduction is permitted which does not comply with these terms.

Marco Massa, MD (凶) marxmassa@gmail.com

Received: 11 May 2020; Accepted: 14 May 2020; Published online: 19 May 2020. doi:10.32044/ijedo.2020.05 


\section{Introduzione}

La diffusione pandemica della malattia da coronavirus (COVID-19) e il conseguente lockdown disposto dai governi, hanno reso più complessa la somministrazione delle terapie psicologiche a tutti i livelli di cura. La necessità di garantire la prosecuzione dei trattamenti per i nostri pazienti con disturbi dell'alimentazione nonostante le limitazioni e le complicazioni cliniche legate all'emergenza COVID-19, alla luce di una sostanziale impossibilità a utilizzare approcci vis a vis (individuali e di gruppo), ha indotto ad implementare nuove strategie per attuare la psicoterapia (Waller, Pugh, Mulkens, et al. 2020). In tal senso la terapia cognitivo comportamentale migliorata (CBT-E) somministrata a distanza (CBT-E online) si sta dimostrando una buona soluzione a questo problema e le esperienze messe in atto in questo periodo a livello di terapia ambulatoriale lo confermerebbero (Murphy, Dalle Grave, et al. 2020).

Questo scritto descrive l'esperienza in corso a Villa del Principe di Genova dove, a partire della fine del 2017 e grazie al contributo di Riccardo Dalle Grave e dell'equipe di Casa di Cura Villa Garda, è stato implementato un programma di trattamento CBT-E residenziale intensivo per i disturbi dell'alimentazione (Dalle Grave, 2017).

In queste settimane di emergenza sanitaria abbiamo dovuto forzatamente applicare rapidamente delle modifiche alle abituali procedure del programma terapeutico senza che ovviamente ci fosse il tempo e il modo di verificarne l'efficacia. Ritengo che nel prossimo futuro queste implementazioni ai protocolli abituali della CBT-E, attraverso l'utilizzo della somministrazione a distanza, possano essere sottoposti a studi approfonditi di efficacia per poterne valutare le possibilità di utilizzo anche al di fuori dell'attuale condizione di emergenza.

Se nell'ambito dei trattamenti ambulatoriali le misure di distanziamento sociale hanno creato un ostacolo alla somministrazione delle psicoterapie, gli effetti della pandemia da COVID-19 hanno avuto un impatto ancora più marcato nei contesti di cura in regime di ricovero nelle strutture di riabilitazione. Durante questa emergenza sanitaria il rischio per una struttura residenziale di diventare un focolaio di contagio è piuttosto elevato. Le misure messe in atto per contrastare tali rischi e per attuarne il contenimento anche attraverso la sospensione o radicale modifica delle abituali attività terapeutico riabilitative, hanno stravolto, nella nostra Unità di Riabilitazione Nutrizionale, l'abituale assetto dei programmi di trattamento previsti dalla CBT-E. Impossibilità di uscire dalla struttura, di ricevere visite di familiari e conoscenti, di fare sedute di terapia di gruppo (o anche individuali vis-a-vis), l'utilizzo dei DPI, la necessità di distanziamento fra le persone, l'isolamento di casi sospetti o confermati di infezione, hanno reso necessario attuare nuove soluzioni per garantire la continuità di cura in modo adeguato.

In modo analogo a quanto avvenuto per i contesti ambulatoriali, si è ritenuto che le caratteristiche specifiche della CBT-E applicata in ambito di ricovero, potessero anch'esse giovarsi di soluzioni "a distanza" per molte procedure del programma terapeutico residenziale e semiresidenziale.

\section{Procedure CBT-E influenzate dalle misure anticontagio COVID-19 adattate con procedure a distanza}

La CBT-E intensiva residenziale utilizza diverse procedure per il proprio programma di trattamento. Alcune di queste sono uguali alla CBT-E ambulatoriale, altre sono specifiche del contesto di cura riabilitativo residenziale (Dalle Grave, 2018). Solo alcune di queste non subiscono l'effetto delle misure attuate per il contenimento del contagio COVID-19 in una struttura di ricovero, in questi casi esse procedono con le modalità abituali. Ad esempio l'automonitoraggio in tempo reale attraverso la compilazione della relativa scheda avviene quasi sempre con le solite modalità attraverso l'utilizzo delle schede cartacee (nella CBT-E ambulatoriale sono allo studio modalità di compilazione delle schede di automonitoraggio su supporto informatico).

La maggior parte delle procedure del programma tuttavia sono influenzate in modo più o meno marcato dai provvedimenti legati al contenimento del contagio. Nella tabella 1 sono elencate le procedure della CBT-E intensiva (in regime di ricovero e pertanto definita anche CBT-E ospedaliera) e come queste subiscono l'impatto dell'emergenza COVID-19.

Descriverò ora più nel dettaglio come abbiamo utilizzato modalità "a distanza" per poter attuare in modo efficace quelle procedure della CBT-E residenziale che hanno subito limitazioni.

Prima di addentrarci in questa descrizione devo precisare un altro aspetto importante del trattamento CBT-E intensivo che ha subito un forte impatto dalle misure anti COVID-19. Mi riferisco alla fase semiresidenziale del trattamento (o di Day Hospital), a quelle sette settimane di trattamento, successivo al ricovero residenziale, durante le quali i pazienti trascorrono in struttura solo la giornata, recandosi poi presso la propria abitazione la sera. Le misure contenute nei provvedimenti del nostro governo hanno 
Tabella 1. Procedure della terapia cognitivo comportamentale migliorata (CBT-E) influenzate dalle misure anticontagio COVID-19 adattate con procedure a distanza

\begin{tabular}{|c|c|c|}
\hline Procedure della CBT-E residenziale & $\begin{array}{l}\text { Influenzata } \\
\text { dalle misure } \\
\text { anticontagio } \\
\text { COVID-19 }\end{array}$ & $\begin{array}{l}\text { Alternative attraverso procedure } \\
\text { di terapia "a distanza" }\end{array}$ \\
\hline Costruire la Formulazione Personalizzata & + & $\begin{array}{l}\text { La creazione o la verifica della formulazione potrebbe } \\
\text { dover essere affrontata in modalità a distanza (in videocall) }\end{array}$ \\
\hline Usare la Scheda di Monitoraggio & 0 & - \\
\hline Misurazione e interpretazione del peso & + & $\begin{array}{l}\text { Misurazione collaborativa del peso invariata. Grafico } \\
\text { del peso condiviso su Zoom. Per le pazienti in DH si } \\
\text { applicano le modalità della CBT-E online ambulatoriale } \\
\text { (la paziente effettua la misurazione durante una video- } \\
\text { chiamata) }\end{array}$ \\
\hline $\begin{array}{l}\text { Valutare l'andamento del trattamento (Eating } \\
\text { Problem Check List) }\end{array}$ & 0 & - \\
\hline Alimentazione assistita & + & $\begin{array}{l}\text { Nessuna alternativa "a distanza". Ci possono essere } \\
\text { problemi legati a esigenze di distanziamento (fra pazienti } \\
\text { e fra pazienti e operatori/dietiste). Pazienti in isolamento } \\
\text { per COVID-19 sospetto o confermato }\end{array}$ \\
\hline Alimentazione non assistita & ++ & $\begin{array}{l}\text { Nessuna alternativa "a distanza". Ci possono essere } \\
\text { problemi legati a esigenze di distanziamento (fra pazienti } \\
\text { e fra pazienti e operatori/dietiste). Pazienti in isolamento } \\
\text { per COVID-19 sospetto o confermato. } \\
\text { I pazienti in DH/semiresidenziale non sono monitorati }\end{array}$ \\
\hline Incontro di revisione settimanale (Tavola Rotonda) & ++ & Viene effettuato in video call (su piattaforma Zoom) \\
\hline Sedute CBT-E individuali & + & $\begin{array}{l}\text { In ricovero non cambiano (distanziamento e DPI o anche } \\
\text { in video call), se caso sospetto/confermato si utilizza } \\
\text { video call. In DH solo in video call (paziente a casa) }\end{array}$ \\
\hline Sedute di gruppo & ++ & $\begin{array}{l}\text { Nelle strutture sanitarie ospedaliere ed extraospedaliere } \\
\text { sono sospese le attività in gruppo. II gruppo si effettua } \\
\text { attraverso video call (Zoom meeting) }\end{array}$ \\
\hline Sedute di attività fisica & +++ & Sono sospese all'interno della struttura \\
\hline CBT-E Familiare (Adolescenti) & +++ & Sospese le sedute con familiari. Si eseguono su video call \\
\hline
\end{tabular}

decretato la sospensione delle attività diurne dei centri di riabilitazione e la chiusura dei centri diurni. Ciò ha costretto ad adottare una soluzione che è apparsa all'inizio un compromesso che temevamo potesse rivelarsi fortemente limitante. Avendo come unica alternativa quella di dimettere la paziente facendole interrompere il programma, abbiamo optato per trasferire integralmente ad una dimensione "a distanza" tutta la fase semiresidenziale del programma, mandando le pazienti a casa e affidandole unicamente ad una terapia online intensiva. Ciò ha sicuramente modificato sostanzialmente le caratteristiche del programma abituale per questa fase. Basti pensare che i pasti, dalla 13 settimana del programma in poi, hanno luogo in questo caso esclusivamente fuori dalla struttura. In sostanza il trattamento semiresidenziale si è trasformato assumendo le caratteristiche di un trattamento ambulatoriale multidisciplinare intensivo somministrato a distanza. Le pazienti partecipano attraverso sistemi di 
videochiamata alle sedute di psicoterapia individuale, alle sedute di gruppo, ai colloqui con le dietiste, alla misurazione collaborativa del peso e all'incontro di revisione settimanale. Nonostante la comprensibile preoccupazione iniziale i risultati avuti finora su una casistica molto limitata sono stati sorprendentemente positivi. Va anche detto che il passaggio dal trattamento in ricovero a quello a intensivo a distanza (con la paziente a casa) rappresenta un "salto" importante nel livello di intensità della cura (maggiore di quello che si verifica normalmente con il passaggio dal ricovero residenziale a quello semiresidenziale). Il "salto" tuttavia risulta molto più graduale quando la paziente termina il programma intensivo a distanza e passa, dopo la dimissione, alla terapia post ricovero (in questo caso il passaggio alla fase ambulatoriale sarà più sfumato rispetto ai programmi abituali).

\section{Sedute CBT-E individuali e costruzione della formulazione personalizzata}

Le sedute di psicoterapia individuale per le pazienti ricoverate sono state fortemente influenzate dalle misure di distanziamento e contenimento del contagio. Le indicazioni sul distanziamento consentirebbero dei colloqui effettuati a distanza di almeno uno-due metri (anche di più vista la durata di 45-50 minuti dell'incontro) e ciò ad esempio rende sostanzialmente impossibile la creazione e lo sviluppo condivisi su un foglio della formulazione personalizzata. A ciò si deve aggiungere che l'utilizzo delle mascherine (necessario) limita molto la comunicazione verbale privandola di gran parte della componente espressiva della mimica facciale. Per queste ragioni la somministrazione on line delle sedute di psicoterapia è parsa opportuna anche all'interno della struttura di ricovero con paziente in una stanza e terapeuta in un altra della stessa struttura (o anche talvolta fuori dalla struttura). La sensazione che abbiamo avuto è che sia più espressiva, e funzionale, la comunicazione attraverso il video a volto scoperto piuttosto che una seduta vis a vis distanziata di un paio di metri e con mascherina indossata da terapeuta e paziente.

Nella CBT-E somministrata online la formulazione transdiagnostica, come già descritto da Murphy et al. (2020), viene creata e mostrata attraverso la telecamera e, a fine seduta, consegnata alla paziente. Ciò non costituisce un problema visto che in questo caso sia paziente che terapeuta sono nella stessa struttura. Alcune piattaforme di videocall (come ad esempio Zoom) consentono inoltre di condividere una lavagna virtuale su cui il terapeuta può disegnare la formulazione.

\section{Scheda di Monitoraggio}

La compilazione della scheda di monitoraggio per le pazienti ricoverate non subisce modifiche. La sua visione in seduta può essere fatta attraverso l'invio di una sua scansione o, se il terapeuta si trova nella struttura, di una semplice fotocopia.

\section{Misurazione e interpretazione del peso}

Per le pazienti ricoverate la procedura della misurazione collaborativa del peso effettuata settimanalmente con l'infermiera si attua con le modalità abituali. Il grafico del peso viene poi condiviso durante la videochiamata dell'incontro di revisione settimanale. Per le pazienti che si trovano a casa propria viene utilizzata la procedura suggerita da Murphy et al. (2020) che consiste nel fare eseguire la misurazione del peso durante una videochiamata e riportare il valore sul grafico sempre in modo condiviso attraverso il video.

\section{Valutare l'andamento del trattamento (Eating Problem Check List)}

La scheda del EPCL viene compilata settimanalmente con le modalità abituali e poi condivisa on line nell'incontro di revisione settimanale in videochiamata. Ad esempio può essere scansionata e posta in condivisione su piattaforma Zoom.

\section{Alimentazione assistita e non assistita}

Sebbene questa procedura non subisca modifiche e si possa svolgere con le modalità consuete, vi possono essere alcune difficoltà legate alle esigenze di distanziamento che richiedono una disposizione dei posti ai tavoli caratterizzata da una maggiore distanza. Le procedure online non vengono coinvolte in questo ambito.

\section{Sedute di gruppo}

Le disposizioni governative legate all'emergenza COVID-19 hanno decretato la sospensione di ogni attività di gruppo all'interno delle strutture sanitarie. Ciò ha reso necessario attivare le sedute con modalità online (attraverso Zoom o Skype) per lo svolgimento dei gruppi psicoeducazionali e dei gruppi di problem solving. Ciò rende fra l'altro possibile la partecipazione alla seduta anche alle pazienti che - come abbiamo visto prima - si trovano a casa loro per svolgere con modalità a distanza la fase semiresidenziale.

\section{Incontro di revisione settimanale (Tavola Rotonda)}

Questa è probabilmente la procedura della CBT-E ospedaliera che più si giova del suo passaggio alla forma online. Tutti i membri dell'equipe si ritrovano in video conferenza assieme alla paziente (a Villa del Principe utilizziamo la 
piattaforma Zoom). Il grafico del peso e la scheda di valutazione dell'andamento settimanale (EPCL) vengono condivise durante il meeting. La paziente, se ricoverata, in genere si trova con l'infermiere o la dietista in una stanza mentre gli altri membri dell'equipe si collegano da remoto ognuno dalla propria postazione (all'interno della struttura $\mathrm{o}$ anche dalla propria abitazione). La paziente che si trova a casa per la fase del programma che corrisponde a quella che prima veniva svolta in modo semiresidenziale, si collega alla conferenza dalla propria abitazione.

\section{Sedute con i familiari (adolescenti)}

Le sedute con i familiari dei pazienti (seduta iniziale e fino a 6 sedute verso la fine del trattamento) previste dalla procedura per gli adolescenti sono effettuate esclusivamente a distanza.

\section{Riunione clinica settimanale}

Non fa parte delle procedure della CBT-E intensiva in senso stretto ma è parte integrante del lavoro dell'equipe multidisciplinare transdiagnostica. Questi incontri - non realizzabili in presenza a causa delle misure di distanziamento - vengono effettuati in videoconferenza con frequenza settimanale (lo stesso giorno in cui si effettuano anche le tavole rotonde on line).

\section{Conclusioni}

Possiamo senz'altro affermare che l'applicazione di procedure di somministrazione a distanza della CBT-E intensiva nella nostra esperienza ha dato risultati che ci hanno sorpreso positivamente dimostrando quanto già evidenziato da altri autori nell'ambito della CBT-E ambulatoriale. Si tratta di osservazioni iniziali su una casistica molto limitata di pazienti che però sembrerebbero confermare che la CBT-E si presta alla somministrazione online. Le sue caratteristiche della CBT-E legate ad un approccio collaborativo che valorizza il ruolo del paziente nella terapia sembrerebbero rinforzare, nella sua somministrazione a distanza, il senso di partecipazione e responsabilizzazione dello stesso nella terapia esaltandone la dimensione di collaborazione alla pari con effetti positivi sull'esito del trattamento che forse vanno a colmare la perdita della vicinanza e presenza fisica abituale in tempi normali.

$\mathrm{Ci}$ è sembrato poi che non tutte le pazienti risultano egualmente inclini alle procedure a distanza. Alcune hanno meno dimestichezza con la tecnologia (quelle meno giovani) mentre per altre pare che l'utilizzo della terapia a distanza metta un po' alla prova il livello di ingaggio nel trattamento. Se una paziente è poco ingaggiata nel trattamento faticherà di più ad utilizzare proficuamente l'approccio a distanza. Potrebbe in tal senso essere utile approfondire questo aspetto anche attraverso uno studio sul vissuto delle pazienti rispetto alla CBT-E online.

È poi emerso anche un altro vantaggio della somministrazione on line delle diverse procedure che è quello di poter effettuare con maggiore facilità e agilità (e minori costi) le supervisioni cliniche attraverso la partecipazione attiva del supervisore alle sedute, alle tavole rotonde alle riunioni di equipe settimanali.

L'esperienza che stiamo facendo, seppur originata da un evento tragico e sconvolgente a livello planetario, sembra aver stimolato la ricerca di soluzioni non preventivate che hanno il valore, se opportunamente verificate, di apportare nuove conoscenze sullo sviluppo della psicoterapia dei disturbi dell'alimentazione.

\section{Ulteriori letture}

Remote CBT for eating disorders. Retrieved from https:// www.babcp.com/Therapists/Remote-CBT-For-ED.aspx.

\section{Bibliografia}

Dalle Grave, R. (2017). Vincere i disturbi dell'alimentazione con il trattamento di Villa Garda. Verona: Positive Press

Dalle Grave, R. (2018). La terapia cognitivo comportamentale multistep dei disturbi dell'alimentazione. Teoria, trattamento e casi clinici. Trento: Erickson

Murphy, R., Calugi, S., Cooper, Z., \& Dalle Grave, R. (2020). Challenges and Opportunities for enhanced cognitive behaviour therapy (CBT-E) in light of COVID-19. The Cognitive Behaviour Therapist, 1-31. doi:10.1017/ S1754470X20000161

Waller, G., Pugh, M., Mulkens, S., Moore, E., Mountford, V. A., Carter, J., . . . Smit, V. (2020). Cognitive-behavioral therapy in the time of coronavirus: Clinician tips for working with eating disorders via telehealth when face-to-face meetings are not possible. International Journal of Eating Disorders. doi:10.1002/eat.23289 